\title{
Drug-Sensitivity and Passive Immunity Mathematical Epidemiological Model for Tuberculosis
}

\section{*DANHAUSA, AA; DANIEL, EE; SHAWULU, CJ; NUHU, AM; PHILEMON, L}

\author{
Department of Mathematics/Computer Science, Federal University, Kashere, Gombe State, Nigeria
}

*Corresponding Author Email: danhausaa@gmail.com

\begin{abstract}
Regardless of many decades of research, the widespread availability of a vaccine and more recently highly visible WHO efforts to promote a unified global control strategy, Tuberculosis remains a leading cause of infectious mortality. In this paper, a Mathematical Model for Tuberculosis Epidemic with Passive Immunity and DrugSensitivity is presented. We carried out analytical studies of the model where the population comprises of eight compartments: passively immune infants, susceptible, latently infected with DS-TB. The Disease Free Equilibrium (DFE) and the Endemic Equilibrium (EE) points were established. The next generation matrix method was used to obtain the reproduction number for drug sensitive $\left(R_{o S}\right)$ Tuberculosis. We obtained the disease-free equilibrium for drug sensitive TB which is locally asymptotically stable when $R_{o s}<1$ indicating that tuberculosis eradication is possible within the population. We also obtained the global stability of the disease-free equilibrium and results showed that the disease-free equilibrium point is globally asymptotically stable when $R_{o s} \leq 1$ which indicates that tuberculosis naturally dies out.
\end{abstract}

\section{DOI:https://dx.doi.org/10.4314/jasem.v25i9.18}

Copyright: Copyright (C) 2021 Danhausa et al. This is an open access article distributed under the Creative Commons Attribution License (CCL), which permits unrestricted use, distribution, and reproduction in any medium, provided the original work is properly cited.

Dates: Received: 09 May 2021; Revised: 12 August 2021; Accepted: 12 September 2021

Keywords: Disease, Tuberculosis, Transmission, Dynamics, Model

Tuberculosis (TB) is a bacterial disease acquired through air-borne infection (Daniel, 2009). Mycobacterium Tuberculosis Complex (MTBC) is the causative agent of tuberculosis. According to the World Health Organization (WHO), one-third of the world's population is infected, either latently or active, with tuberculosis (WHO, 2016). It is an ancient disease with evidence of its existence being found in relics from ancient Egypt, India and China. In the eighteenth century, Western Europe suffered terribly from this disease with prevalence as high as 900 deaths per 100,000 . This was largely due to poor ventilation, overcrowded housing, primitive sanitation and malnutrition among other risk factors that led to the epidemic (Daniel, 2009). Tuberculosis is spread through the air from one person to another. The bacteria get into the air when someone who has a tuberculosis lung infection coughs, sneezes, shouts, or spits. People who are nearby can then possibly breathe the, bacteria into their lungs and become infected. Even though the disease is airborne, it is believed that TB is not highly infectious and so, occasional contacts with infectious person rarely lead to infection. TB cannot be spread through handshakes, sitting on toilet seats or sharing dishes and utensils with someone who has TB (Abdul-halim, 2013).

*Corresponding Author Email: danhausaa@gmail.com
TB is the ninth leading cause of death worldwide and the leading cause from a single infectious agent, ranking above HIV/AIDS (WHO, 2017). In 2016, there were an estimated 1.3 million TB deaths among HIV-negative people (down from I .7 million in 2000) and an additional 374,000 deaths among HIV-positive people. An estimated 10.4 million people fell ill with TB in 2016: $90 \%$ were adults, $65 \%$ were male, $10 \%$ were people living with HIV (74\% in Africa) and 56\% were in five countries: India, Indonesia, China, the Philippines and Pakistan (WHO, 2017). DrugResistant TB is a continuing threat, in 2016, there were 600, 000 new cases with Resistance to Rifampicin (RR-TB), the most effective first-line drug, of which 490, 000 had Multidrug-Resistant TB (MDR-TB). Almost half (47\%) of these cases were in India, China and the Russian Federation (WHO, 2017). Tuberculosis (TB) is a preventable disease linked to poverty, was declared an emergency in Africa in 2005. Each year it claims the lives of half a million Africans, many young and in their most productive years. In the past 15 years, overall rates have doubled in Africa and tripled in high HIV areas. Africa has the highest per capital incidence $0.1 \mathrm{~TB}$ in the world $(28 \%)$, with most of 15 the worst affected countries located in subSaharan Africa. Those most at risk include the urban poor, migrants and refugees, who are forced to live in 
overcrowded conditions (Ibrahim, et al, 2013). Africa is also the only continent where TB rates are increasing, with 1,500 TB deaths every day. Tragically and avoidably, $10 \%$ of these are children. TB is also a leading killer of HIV-positive people with weakened immune systems. About 200,000 people living with HIV/AIDS die from TB every year, most of them in Africa. Completing a particularly vicious circle, HIV itself has been the single most important factor in the rising incidence of TB in Africa since 1990. Treating co-infected people is hard as the drug therapies for each are hard to safely combine (Ibrahim, et al., 2013). However, the world is still far from defeating the disease. About 8 billion US dollars per year is needed for a full response to the global tuberculosis epidemic in low and middle income countries by the year 2025 with a funding gap of 2.3 billion US dollars per year. This amount excluded resources required for research and development, which was estimated to be about 2 billion US dollars yearly (WHO, 2017). Clearly, this reveals that the current investment in tuberculosis falls below the low and middle-income country's needs. (MTB) bacteria spread through inhaling droplets from the cough or sneeze of a person suffering from active tuberculosis (WHO, 2017). The bacteria enter the body causing a MTB infection affecting majorly the lungs but it can also affect any other part of the body including the urinary tract, brain, lymph nodes, bones, joints and the ear. Person(s) with lowered immunity such as those with HIV, diabetes, immune disorders, end-stage renal disease, those on drugs that suppress immunity, young children and pregnant women among others are at a higher risk of contracting the disease (WHO, 2017). Population movements have significant implications for tuberculosis transmission as migration introduces tuberculosis problem to the areas to which the migrants migrate to. Temporary migrant workers often bring the bacteria to lower prevalence areas and local transmission can be readily established (Semenza, et al., 2010). Tuberculosis is curable provided an early diagnosis is made and one follows the proper treatment regimen which could take six months up to two years for the active tuberculosis to clear (Trauer, et al., 2014).. In 2016, there were an estimated 480. 000 new cases of Multidrug-Resistant TB (MDR-TB) and an additional 100, 000 people with Rifampicin-Resistant TB (RR-TB) who were also newly eligible for MDR-TB treatment. India, China and the Russian Federation accounted for $45 \%$ of the combined total of 580, 000 cases (WHO, 2017). Despite many decades of study, the widespread availability of a vaccine and more recently highly visible WHO efforts to promote a unified global control strategy, TB remains a leading cause of infectious mortality (WHO, 2017). Recent data indicate that the overall global incidence of TB is rising as a result of resurgence of the disease in Africa, parts of Eastern Europe and Asia (WHO, 2017). In 2016, there were an estimated L3 million TB deaths among HIV-negative people (down from 1.7 million in 2000) and an additional 374, 000 deaths among HIV-positive people. An estimated 10.4 million people fell ill with TB in 2016: $90 \%$ were adults, $65 \%$ were male, $10 \%$ were people living with HIV (74\% in Africa) and $56 \%$ were in five countries: India, Indonesia, China, the Philippines and Pakistan (WHO, 2017). Waaler (1968) formulated a deterministic model for the transmission dynamics of tuberculosis. However, the model did not incorporate passive immunity, drug-sensitive 'I'B and drug-resistant TB. In view of this, we modified the model to investigate the effects of Passive Immunity, Drug-Sensitive and Drug-Resistance on transmission dynamics of tuberculosis. The aim of this study is to mathematically model the effects of Passive immunity and drug-sensitive on the transmission dynamics of tuberculosis.

\section{MODEL NOTATION}

TB Model (Waaler, 1968): We reviewed the existing model by Waaler, (1968) in terms of assumptions, model description, and model equations. This serves as a framework for the model with passive immunity and drug sensitivity TB on the transmission dynamic of tuberculosis.

TB Model Assumptions: The model is based on the following assumptions

i. Age, sex, social status, race occupied with climatic conditions in the district does not affect the probability of an individual being infected.

ii. The disease is transmitted in a close environment. There is no emigration or immigration.

ii. Susceptible individuals are moving to the infected class.

iv. Individuals either die by infection or natural death

v. It is also assumed that infected individuals either die or recover.

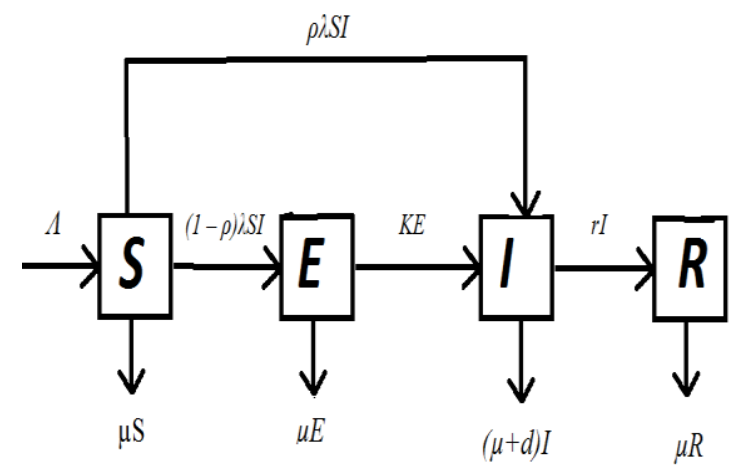

Fig. 1: Schematic sDiagram of Existing Model 
Description of the TB Model: In the model developed by Waaler (1968), the population is divided into four classes: susceptible class $\mathrm{S}(\mathrm{t})$, exposed $\mathrm{E}(\mathrm{t})$, infected $\mathrm{I}(\mathrm{t})$, and recovered $\mathrm{R}(\mathrm{t})$. The class of susceptible individuals $\mathrm{S}$ is increased by birth rate $\Lambda$. The class reduces due to the progression of individuals to the infectious class at the rate $\rho \lambda S I$, due to the progression of the individuals to the exposed class grows as a result of incoming of individuals at the rate $(1-\rho) \lambda S I$ and as a result of natural death $\mu \mathrm{S}$. The exposed class grows as a result of incoming individuals from susceptible class at the rate $(1-\rho) \lambda S I$, the class reduces as a result of progression of individuals to the infectious class at the rate $K E$ and reduces due to the rate of natural death at the rate $\mu E$.

The infectious class grows as a result of incoming individuals from the expose class at the rate $\mathrm{KE}$ and also as a result of coming in of individuals from the susceptible class at the rate $\rho \lambda S I$. the class reduces due to the progression of individuals to the recovered class at the rate $r I$, due to the TB mortality rate $\mathrm{dI}$ and due to the natural death at the rate $\mu I$. the recovered class grows as a result of successful treatment and cure of in infectious individuals at the rate and reduces as a result of natural death at the rate $\mu R$.

Table 1: Variables and Parameters of the TB Model (Waaler, 1968)

\begin{tabular}{ll}
\hline VAR/PAR & PAR DESCRIPTION \\
\hline$S(t)$ & $\begin{array}{l}\text { the number of susceptible individuals at time } \\
t .\end{array}$ \\
& the number of latently infected/ exposed \\
& individuals at time $t$. \\
& the number of infected individual at time $t$. \\
$R(t)$ & the number of recovered individuals at time $t$ \\
$\Lambda$ & the recruitment number in the population. \\
$\rho$ & the proportion of the new infectious that move \\
$\mu$ & directly into the infected class. \\
$k$ & the natural mortality rate. \\
$r$ & the reactivation rate. \\
$d$ & the recovery rate. \\
$\lambda$ & the TB mortality rate. \\
& The force of infection
\end{tabular}

\section{Existing Model Equations}

$$
\begin{aligned}
& \frac{d s}{d t}=\Lambda-\lambda(I) S-\mu S \\
& \frac{d E}{d t}=(1-\rho) \lambda(I) S-(\mu+K) E \\
& \frac{d I}{d t}=\rho \lambda(I) S+K E-(\mu+d+r) I \\
& \frac{d R}{d t}=r I-\mu R
\end{aligned}
$$

Model with Passive Immunity and Drug- Sensitivity $T B$ : Below are the assumptions, description, diagram and model equations of the formulated modified model.
Additional Assumptions for the Model with Passive Immunity and Drug-Sensitivity TB: The population is heterogeneous. That is, the individual that make up the population can be grouped into different compartment or classes according to their epidemiological state.

i. It is assumed that the only way of entry into the population is through birth or new born babies and the only way of exist is via death from the natural causes or death from TB related causes

ii. All newborns are previously uninfected by TB and therefore join either immunized compartment or the susceptible compartment depending on weather they vaccinated or not

iii. The vaccinated individuals do not acquire permanent immunity

iv. The effective treatment rate of drugsensitivity is higher than that of drug-resistant

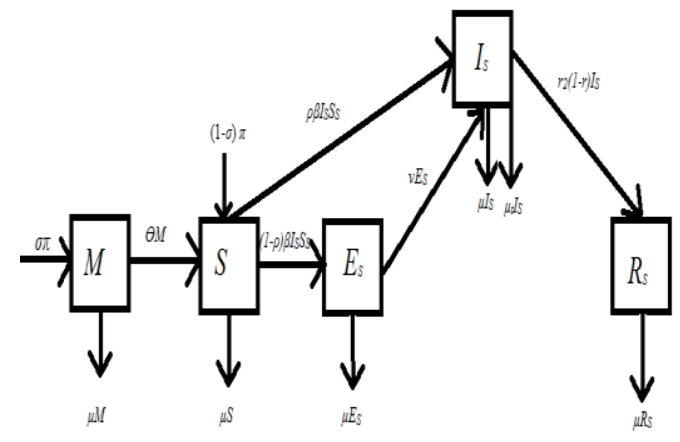

Fig. 2: Schematic drawing for the Model with Passive Immunity and Drug Sensitivity TB

Description of the Model with Passive Immunity and Drug Sensitivity TB: Based on the standard MSEIR Model, the population is partitioned into five (5) compartment or classes namely; passive immunized infants $M(t)$, susceptible $S(t)$, latently infected individuals with drug-sensitivity $\mathrm{TB} \mathrm{E}_{\mathrm{s}}(\mathrm{t})$, infectious individuals with drug sensitivity $\mathrm{TB} \mathrm{I}_{\mathrm{s}}(\mathrm{t})$, recovered individuals with drug sensitivity $T B \mathrm{R}_{\mathrm{s}}(\mathrm{t})$. The passive immunized compartment increase due to the coming in of the immunized newborns into the population, where we assume that a population, $\sigma \pi$ of the incoming individuals are immunized through vaccination. The compartment reduces due to the progression of individuals in this class to the susceptible class as a result of the expiration of the duration of vaccine efficiency at the rate of $\theta M$ and also as a result of natural death at the rate $\mu M$. The susceptible compartment of the population grows due to the coming in of new born babies not immunized against TB infection into the population at the rate $(1-\sigma) \pi$ and as a result of the expiration of the efficiency of the vaccine at the rate $\theta M$. This compartment decreases due to the progression of individuals into the latently infected individuals with drug-sensitivity $\mathrm{TB}$ at the 
rate $(1-\rho) \beta I_{S} S_{S}, \quad$ infectious individuals with drugsensitivity at the rate $\rho \beta I_{S} S_{S}$, and also as a result of natural death at the rate of $\mu S$. The population of latently infected individuals with drug-sensitivity grows as a result of progression of individuals from the susceptible class at the rate $(1-\rho) \beta I_{S} S_{S}$. This class reduces due to the progression of latently infected individuals with drug-sensitivity TB at the rate $v E_{S}$ and as a result of death from natural causes at the rate $\mu E_{S}$. The population of the infectious individuals with drugsensitivity TB grows due to the progression of latently infected individuals with drug-sensitivity at the rate $v E_{S}$ and due to the progression of susceptible individuals at the rate $\rho \beta I_{S} S_{S}$. this compartment reduces due to the progression of recovered individuals with drug-sensitivity at the rate $r_{2}(1-r) I_{S}$ due to the death as a result of active TB with drugsensitivity at the rate $\mu_{t} I_{S}$ and also as a result of death from natural causes at the rate $\mu I_{S}$. The recovered compartment with drug-sensitivity TB grows as a result of successful treatment and cure of infectious with drug-sensitivity at the rate $r_{2}(1-r) I_{S}$ and reduces as a result of death from natural causes at the rate $\mu R_{S}$.

Table 2: Parameter and Variable of the Model with Passive Immunity and Drug-Sensitivity TB

\begin{tabular}{|c|c|}
\hline VAR / PAR & DESCRIPTION \\
\hline $\mathrm{M}(\mathrm{t})$ & $\begin{array}{l}\text { the number of people who were immunized } \\
\text { against } T B \text { through vaccination at a time } t \text {. }\end{array}$ \\
\hline$S(t)$ & $\begin{array}{l}\text { The number of susceptible individuals at a time } \\
\text { t. }\end{array}$ \\
\hline $\mathrm{E}_{\mathrm{s}}(\mathrm{t})$ & $\begin{array}{l}\text { The number of latently infected individual with } \\
\text { drug-sensitivity TB at a time t. }\end{array}$ \\
\hline$I_{s}(t)$ & $\begin{array}{l}\text { the number of infectious individuals with drug- } \\
\text { sensitivity TB at time t }\end{array}$ \\
\hline $\mathrm{R}_{\mathrm{s}}(\mathrm{t})$ & $\begin{array}{l}\text { The number of recovered individuals with } \\
\text { drug-sensitivity } \mathrm{TB} \text { at a time t. }\end{array}$ \\
\hline $\begin{array}{l}\beta_{S} \\
\pi\end{array}$ & $\begin{array}{l}\text { the transmission rate of drug-sensitivity TB } \\
\text { the recruitment rate }\end{array}$ \\
\hline$r_{1}$ & The treatment efficiency of drug-sensitivity TB \\
\hline$\sigma$ & $\begin{array}{l}\text { The proportion of newborn that have been } \\
\text { immunized through immunization. }\end{array}$ \\
\hline$\theta$ & The rate of expiration of vaccination. \\
\hline$\mu$ & natural mortality rate \\
\hline $\mathrm{V}$ & $\begin{array}{l}\text { progression rate for latent TB to active TB for } \\
\text { drug-sensitivity cases }\end{array}$ \\
\hline$\mu$ & the mortality rate due to $\mathrm{TB}$ \\
\hline $\mathrm{P}$ & $\begin{array}{l}\text { the proportion of new infections that produces } \\
\text { active TB for drug-sensitivity cases }\end{array}$ \\
\hline
\end{tabular}

Model Equation with Passive Immunity and DrugSensitivity $T B$

$$
\begin{aligned}
& \frac{d M}{d t}=\sigma \pi-(\theta+\mu) M \\
& \frac{d s}{d t}=(1-\sigma) \pi+\theta M-(\beta \mathrm{s} I \mathrm{~s}+\mu) S \\
& \frac{d E \mathrm{~s}}{d t}=(1-\rho \mathrm{s}) \beta \mathrm{s} I \mathrm{~s} S-(\mathrm{v}+\mu) E \mathrm{~s} \\
& \frac{d \mathrm{~s}}{d t}=\rho \mathrm{s} \beta \mathrm{s} I \mathrm{~s} S+\mathrm{v} E \mathrm{~S}-(\mu+\mu \mathrm{T}+r 2) I \mathrm{~s} \\
& \frac{d R \mathrm{~s}}{d t}=r 2(1-\mathrm{r}) I \mathrm{~s}-\mu \mathrm{Rs}
\end{aligned}
$$

Where

$\mathrm{M}(0)=\mathrm{M} 0, \mathrm{~S}(0)=\mathrm{S} 0$, Es $(0)=\mathrm{E} 0$, Is $(0)=\mathrm{I} 0$, Rs $(0)$ $=\mathrm{R} 0$.

Method of Model Analysis: In this section, various methods adopted in carrying out this study were discussed:

Equilibrium State: The equilibrium state for the model is obtained by setting the model equations to be zero. i.e

$$
\frac{d M}{d t}=\frac{d S}{d t}=\frac{d E S}{d t}=\frac{d I S}{d t}=\frac{d R S}{d t}=0
$$

Basic Reproduction Number: Dieckmann and Heesterbeek (2000) defined the basic reproduction number, $R_{0}$ as the average number of secondary infections caused by an infectious individual during his/her entire life as an infectious person. In this model, we adopted the model of the next generation matrix to compute our reproduction number. We call $\mathrm{FV}^{-1}$ the next generation matrix for the model and set the reproduction number $\mathrm{R}_{0}=\rho\left(\mathrm{FV}^{-1}\right)$ where $\mathrm{F}=\left(\frac{\partial F \mathrm{i}(x 0)}{\partial x \mathrm{j}}\right)$ and $\mathrm{V}=\left(\frac{\partial V \mathrm{i}(x 0)}{\partial x \mathrm{j}}\right)$ for $\mathrm{i} \geq 1$ the number of compartments and $1 \leq \mathrm{j} \leq \mathrm{m}$ for the infected compartments only. $\rho\left(\mathrm{FV}^{-1}\right)$ denotes the spectral radius of the matrix $\mathrm{A}$. $\mathrm{F}$ and $\mathrm{V}$ are $\mathrm{m} \times \mathrm{m}$ matrices, where $m$ is the number of infected classes (Dieckmann and Heesterbeek 2000).

Local Stability Analysis of the Disease Free Equilibrium State: We use Routh-Hurwitz criterion to obtain the steady state of the model. The RouthHurwitz criterion states that a necessary and sufficient condition that the equation $\mathrm{x}^{\mathrm{n}}+\mathrm{a}_{1} \mathrm{x}^{\mathrm{n}-1}+\ldots+\mathrm{a}_{\mathrm{n}}=0$, (with real coefficients) have only roots of negative real part if the values of the determinants of the matrices are all positive, D1=a1 $>0, \mathrm{D} 2=\begin{array}{cc}a_{1} & a_{3} \\ 1 & a_{2}\end{array}>0$, where

$\begin{array}{ccc}a 1 & a & a 5 \\ \mathrm{D} 3= & a 2 & a 4 \\ 0 & a & a 3\end{array}$

$\mathrm{Dk}=\left[\begin{array}{ccccc}a 1 & a 3 & . & . & 0 \\ 1 & a 2 & a 4 & . & . \\ 0 & a 1 & a 3 & . & . \\ 0 & 1 & a 2 & . & . \\ . & . & & . & . \\ 0 & 0 & & . & a \mathrm{k}\end{array}\right]>0 \quad$ Called the Hurwitz matrix

For quadratic and cubic polynomials, these conditions reduce to:

$n=2, \mathrm{a} 1>0, \quad \mathrm{a} 2>0$ 
$n=3, \mathrm{a} 1>0 \quad \mathrm{a} 2>0 \quad \mathrm{a} 1 \mathrm{a} 2>0$.

\section{RESULTS AND DISCUSSION}

Analytic Results: We developed a mathematical model for the effect of passive immunity and drug-sensitivity on the transmission dynamic of tuberculosis. The model equations are (5) to (9)

Disease Free Equilibrium State: The equilibrium state for the system was obtained by setting the model equation to zero. i.e.

$\frac{d M}{d t}=\frac{d S}{d t}=\frac{d E S}{d t}=\frac{d I S}{d t}=\frac{d R S}{d t}=0$

Thus, at equilibrium, equation (5) to (9) becomes

$$
\begin{aligned}
& \sigma \pi-(\theta+\mu) M=0 \\
& (1-\sigma) \pi+\theta M-(\beta s I s+\mu) S=0 \\
& (1-\rho s) \beta s I s S-(\mathrm{v}+\mu) E s=0 \\
& \rho s \beta s I s S+v E s-(\mu+\mu t+r 2) I s=0 \\
& r 2(1-r) I s-\mu R s=0
\end{aligned}
$$

At disease free,

$E \mathrm{~s}=0, I \mathrm{~s}=0, \mathrm{Rs}=0$

Substituting equation (16) into equation (11), we have

$\mathrm{M}=\frac{\sigma \pi}{\theta+\mu}$

Substituting equation (17) into equation (12), we have
Therefore, the disease-free equilibrium state is

$\mathrm{E} 0=(\mathrm{M}, \mathrm{S}, \mathrm{Es}, \mathrm{Is}, \mathrm{Rs})=\frac{\sigma \pi}{\theta+\mu}, \frac{\pi(\theta+\mu-\mu \sigma)}{\mu(\theta+\mu)}, 000$ (19)

Endemic Equilibrium State: The endemic equilibrium point is the point at which the disease persists in a given population

From equation (11), we have

$\sigma \pi-(\theta+\mu) M=0$

$\mathrm{M}=\frac{\sigma \pi}{\theta+\mu}$

Substituting equation (20) in (12), we have

$$
\begin{aligned}
& (1-\sigma) \pi+\theta \frac{\sigma \pi}{\theta+\mu}-(\beta \mathrm{s} I \mathrm{~s}+\mu) S=0 \\
& \mathrm{~S}=\frac{(\theta+\mu)(1-\sigma) \pi+\theta \sigma \pi}{(\theta+\mu)(\beta \mathrm{s} I \mathrm{~s}+\mu)}
\end{aligned}
$$

Substituting equation (22) into equation (13), we have

$$
\begin{aligned}
& (1-\rho \mathrm{s}) \beta \mathrm{s} I \mathrm{~s} \frac{(\theta+\mu)(1-\sigma) \pi+\theta \sigma \pi}{(\theta+\mu)(\beta \mathrm{s} I \mathrm{~s}+\mu)}-(\mathrm{u}+ \\
& \mu) E \mathrm{~S}=0 \quad(23) \\
& E \mathrm{~S}=\frac{(1-\rho \mathrm{S}) \beta \mathrm{SIS}(\theta+\mu)(1-\sigma) \pi+\theta \sigma \pi}{(\theta+\mu)(\mathrm{v}+\mu)(\beta \mathrm{s} I \mathrm{~s}+\mu)}
\end{aligned}
$$

Substituting equation (24) into equation (14), we have

$\mathrm{S}=\frac{\pi(\theta+\mu-\mu \sigma)}{\mu(\theta+\mu)}$

$\rho \mathrm{s} \beta \mathrm{s} I \mathrm{~s} S+\mathrm{v} \frac{(1-\rho \mathrm{s}) \beta \mathrm{s} I \mathrm{~s}(\theta+\mu)(1-\sigma) \pi+\theta \sigma \pi}{(\theta+\mu)(\mathrm{v}+\mu)(\beta \mathrm{s} I \mathrm{~s}+\mu)}-(\mu+\mu \mathrm{T}+r 2) I \mathrm{~s}=0$

$I S=\frac{v(1-\rho \operatorname{S}) \beta \operatorname{SIS}(\theta+\mu)(\beta \mathrm{s} I \mathrm{~s}+\mu) \rho \operatorname{S} \beta \operatorname{SISS}(\theta+\mu)(1-\sigma) \pi+\theta \sigma \pi}{(\theta+\mu)(v+\mu)(\beta \mathrm{s} I \mathrm{~s}+\mu)(\theta+\mu)(\mu+\mu \mathrm{T}+r 2)(\beta \mathrm{s} I \mathrm{~s}+\mu)-\rho \operatorname{S} \beta \mathrm{S}(\theta+\mu)(1-\sigma) \pi+\theta \sigma \pi}$

$I \mathrm{~s}=\mathrm{A}$

Substituting equation (26) into (15), we have

$r 2(1-r) A-\mu R s=0$

$\mathrm{Rs}=\frac{r 2(1-\mathrm{r}) A}{\mu}$

Hence the endemic equilibrium point of the model $(M, S, E \mathrm{~S}, I S$, RS $)$ is expressed as follows:

$\mathrm{M}=\frac{\sigma \pi}{\theta+\mu}$ 


$$
\begin{aligned}
& \mathrm{S}=\frac{(\theta+\mu)(1-\sigma) \pi+\theta \sigma \pi}{(\theta+\mu)(\beta \mathrm{s} I \mathrm{~s}+\mu)} \\
& E \mathrm{~S}=\frac{(1-\rho \mathrm{s}) \beta \mathrm{s} I \mathrm{~s}(\theta+\mu)(1-\sigma) \pi+\theta \sigma \pi}{(\theta+\mu)(\mathrm{v}+\mu)(\beta \mathrm{s} I \mathrm{~s}+\mu)} \\
& I \mathrm{~s}=\frac{\mathrm{v}(1-\rho \mathrm{s}) \beta \mathrm{s} I \mathrm{~s}(\theta+\mu)(\beta \mathrm{s} I \mathrm{~s}+\mu) \rho \mathrm{S} \beta \mathrm{s} I \mathrm{~s} S(\theta+\mu)(1-\sigma) \pi+\theta \sigma \pi}{(\theta+\mu)(\mathrm{v}+\mu)(\beta \mathrm{s} I \mathrm{~s}+\mu)(\theta+\mu)(\mu+\mu \mathrm{T}+r 2)(\beta \mathrm{s} I \mathrm{~s}+\mu)-\rho \mathrm{s} \beta \mathrm{s}(\theta+\mu)(1-\sigma) \pi+\theta \sigma \pi}
\end{aligned}
$$

$\mathrm{Rs}=\frac{r 2(1-\mathrm{r}) A}{\mu}$

Reproduction Number for Drug-Sensitivity TB: The basic reproduction number of drug-sensitivity is denoted by $\mathrm{R}_{0 \mathrm{~S}}$. It is an important parameter that is used in studying the behavior of epidemiological model. It is defined as the average number of secondary infections infected by an infective individual during an infective period provided that the all members of the population are susceptible. It is an important threshold parameter that determines whether or not, an infection will spread through a given population. We apply the next generation matrix technique by Diekman and Heesterbeek (2000) to obtain the basic reproduction number for drugsensitivity, $R_{0 S}$ by considering the infected compartment of the system (5) to (9) that is equation (7) and (8). Let $F_{1}$ be the rate of appearance of new infection in the $I$ compartment and $\mathrm{V}_{1}$ be the rate of transfer of individuals out of $i$. given the disease free equilibrium, then $\mathrm{R}_{0 \text { s }}$ spectral radius (largest Eigen values) of the next generation matrix denoted by $G=F V^{-1}$

Let $\mathrm{x}=\left(\mathrm{E}_{\mathrm{S}}, \mathrm{I}_{\mathrm{S}}\right)^{\mathrm{T}}$, so that $\frac{d x}{d t}=F 1(\mathrm{X})-V 1(X)$, where

$\mathrm{F}_{1}(\mathrm{X})=\left(\begin{array}{c}\mathrm{F} 1 \\ F 2\end{array}\right)=\left(\begin{array}{c}(1-\rho \mathrm{S} \beta \mathrm{S} I \mathrm{~S}) \\ \rho \mathrm{S} \beta \mathrm{S} I \mathrm{~S}\end{array}\right)$

Evaluating the Jacobean matrix of $\mathrm{F}(\mathrm{X})$

$$
\frac{\partial F 1}{\partial X 2}=\left[\begin{array}{ll}
\frac{\partial F 1}{\partial E S} & \frac{\partial F 1}{\partial I S} \\
\frac{\partial F 2}{\partial E S} & \frac{\partial F 2}{\partial I S}
\end{array}\right]
$$

Substituting equation (30) into equation (31) and

$$
\mathrm{F}=\left[\begin{array}{cc}
0 & \frac{(1-\rho \mathrm{S}) \beta \mathrm{S} \pi(\theta+\mu-\mu \sigma)}{\mu(\theta+\mu)} \\
0 & \frac{\rho \mathrm{S} \beta \mathrm{S} \pi(\theta+\mu-\mu \sigma)}{\mu(\theta+\mu)}
\end{array}\right]
$$

And

$\mathrm{V}_{1}(\mathrm{x})=\left(\begin{array}{c}\mathrm{V} 1 \\ \mathrm{~V} 2\end{array}\right)=\left(\begin{array}{c}(\mathrm{v}+\mu) E \mathrm{~S} \\ (\mu+\mu \mathrm{T}+r 2) I \mathrm{~S}-\mathrm{v} E \mathrm{~S}\end{array}\right)$

Evaluating the Jacobean matrix of $\mathrm{V}(\mathrm{x})$

$\frac{\partial V 1}{\partial X 2}=\left[\begin{array}{ll}\frac{\partial V 1}{\partial E S} & \frac{\partial V 1}{\partial I S} \\ \frac{\partial V 2}{\partial E S} & \frac{\partial V 2}{\partial I S}\end{array}\right]$

Substituting equation (33) into equation (34) and evaluating at disease free equilibrium $\mathrm{E}_{0}$, we obtained $\mathrm{V}=\left[\begin{array}{cc}(v+\mu) & 0 \\ -\mathrm{v} & (\mu+\mu \mathrm{T}+r 2)\end{array}\right]$

Thus, we evaluate equation (35) to get

$\operatorname{det}(\mathrm{V})=(\mathrm{v}+\mu)(\mu+\mu \mathrm{T}+r 2)$

and

$\operatorname{adj}(\mathrm{V})=\left[\begin{array}{cc}(\mu+\mu \mathrm{T}+r 2) & 0 \\ \mathrm{v} & (\mathrm{v}+\mu)\end{array}\right]$

Hence,

$$
\mathrm{V}^{-1}=\left[\begin{array}{cc}
\frac{1}{(v+\mu)} & 0 \\
\frac{1}{(v+\mu)(\mu+\mu \mathrm{T}+r 2)} & \frac{1}{(\mu+\mu \mathrm{T}+r 2)}
\end{array}\right]
$$
evaluating at disease free equilibrium $\mathrm{E}_{0}$, we obtain

Hence, we obtain the matrix $G=F V^{-1}$ by multiplying equation (32) and equation (38) to obtain

$\mathrm{FV} \mathrm{V}^{-1}=\left[\begin{array}{cc}\frac{\mathrm{v}(1-\rho \mathrm{S}) \beta \mathrm{S} \pi(\theta+\mu-\mu \sigma)}{\mu(\theta+\mu)(\mathrm{v}+\mu)(\mu+\mu \mathrm{T}+r 2)} & \frac{(1-\rho \mathrm{S}) \beta \mathrm{S} \pi(\theta+\mu-\mu \sigma)}{\mu(\theta+\mu)(\mathrm{v}+\mu)(\mu+\mu \mathrm{T}+r 2)} \\ \frac{v \rho \mathrm{S} \beta \mathrm{S} \pi(\theta+\mu-\mu \sigma)}{\mu(\theta+\mu)(\mathrm{v}+\mu)(\mu+\mu \mathrm{T}+r 2)} & \frac{(1-\rho \mathrm{S}) \beta \mathrm{S} \pi(\theta+\mu-\mu \sigma)}{\mu(\theta+\mu)(\mu+\mu \mathrm{T}+r 2)}\end{array}\right]$

Therefore, we evaluate the characteristic equation $\left|F^{-1}-\lambda I\right|=0$ of equation (39) to get 
$\left[\begin{array}{cc}\frac{v(1-\rho S) \beta S \pi(\theta+\mu-\mu \sigma)}{\mu(\theta+\mu)(v+\mu)(\mu+\mu \mathrm{T}+r 2)}-\lambda & \frac{(1-\rho \mathrm{S}) \beta \mathrm{S} \pi(\theta+\mu-\mu \sigma)}{\mu(\theta+\mu)(\mathrm{v}+\mu)(\mu+\mu \mathrm{T}+r 2)} \\ \frac{v \rho \mathrm{S} \beta \mathrm{S} \pi(\theta+\mu-\mu \sigma)}{\mu(\theta+\mu)(\mathrm{v}+\mu)(\mu+\mu \mathrm{T}+r 2)} & \frac{(1-\rho \mathrm{S}) \beta \mathrm{S} \pi(\theta+\mu-\mu \sigma)}{\mu(\theta+\mu)(\mu+\mu \mathrm{T}+r 2)}-\lambda\end{array}\right]=0$

$\left(\frac{\mathrm{v}(1-\rho \mathrm{S}) \beta \mathrm{S} \pi(\theta+\mu-\mu \sigma)}{\mu(\theta+\mu)(\mathrm{v}+\mu)(\mu+\mu \mathrm{T}+r 2)}-\lambda\right)\left(\frac{(1-\rho \mathrm{S}) \beta \mathrm{S} \pi(\theta+\mu-\mu \sigma)}{\mu(\theta+\mu)(\mu+\mu \mathrm{T}+r 2)}-\lambda\right)-\left(\frac{\mathrm{v} \rho \mathrm{S} \beta \mathrm{S} \pi(\theta+\mu-\mu \sigma)}{\mu(\theta+\mu)(\mathrm{v}+\mu)(\mu+\mu \mathrm{T}+r 2)}\right)=0$

$\lambda^{2}-\lambda\left[\frac{\mathrm{v}(1-\rho \mathrm{S}) \beta \mathrm{S} \pi(\theta+\mu-\mu \sigma)}{\mu(\theta+\mu)(\mathrm{v}+\mu)(\mu+\mu \mathrm{T}+r 2)}+\frac{(1-\rho \mathrm{S}) \beta \mathrm{S} \pi(\theta+\mu-\mu \sigma)}{\mu(\theta+\mu)(\mu+\mu \mathrm{T}+r 2)}\right]=0$

Hence, simplifying (41) yields

$\lambda=\frac{\left(\frac{v(1-\rho \mathrm{S}) \beta \mathrm{S} \pi(\theta+\mu-\mu \sigma)}{\mu(\theta+\mu)(\mathrm{v}+\mu)(\mu+\mu \mathrm{T}+r 2)}+\frac{\rho \mathrm{S} \beta \mathrm{S} \pi(\theta+\mu-\mu \sigma)}{\mu(\theta+\mu)(\mathrm{v}+\mu)(\mu+\mu \mathrm{T}+r 2)}\right) \pm \sqrt{\left(\frac{\mathrm{v}(1-\rho \mathrm{S}) \beta \mathrm{S} \pi(\theta+\mu-\mu \sigma)}{\mu(\theta+\mu)(\mathrm{v}+\mu)(\mu+\mu \mathrm{T}+r 2)}+\frac{\rho \mathrm{S} \beta \mathrm{S} \pi(\theta+\mu-\mu \sigma)}{\mu(\theta+\mu)(\mathrm{v}+\mu)(\mu+\mu \mathrm{T}+r 2)}\right)^{2}}}{2}$

$\lambda=\frac{\left(\frac{v(1-\rho S) \beta S \pi(\theta+\mu-\mu \sigma)}{\mu(\theta+\mu)(v+\mu)(\mu+\mu \mathrm{T}+r 2)}+\frac{\rho \mathrm{S} \beta \mathrm{S} \pi(\theta+\mu-\mu \sigma)}{\mu(\theta+\mu)(\mathrm{v}+\mu)(\mu+\mu \mathrm{T}+r 2)}\right) \pm\left(\frac{\mathrm{v}(1-\rho \mathrm{S}) \beta \mathrm{S} \pi(\theta+\mu-\mu \sigma)}{\mu(\theta+\mu)(\mathrm{v}+\mu)(\mu+\mu \mathrm{T}+r 2)}+\frac{\rho \mathrm{S} \beta \mathrm{S} \pi(\theta+\mu-\mu \sigma)}{\mu(\theta+\mu)(\mathrm{v}+\mu)(\mu+\mu \mathrm{T}+r 2)}\right)^{2}}{2}$

Hence, $\lambda_{1}=0 ; \lambda_{2}=\frac{v(1-\rho S) \beta S \pi(\theta+\mu-\mu \sigma)}{\mu(\theta+\mu)(v+\mu)(\mu+\mu \mathrm{T}+r 2)}+\frac{\rho \mathrm{S} \beta \mathrm{S} \pi(\theta+\mu-\mu \sigma)}{\mu(\theta+\mu)(v+\mu)(\mu+\mu \mathrm{T}+r 2)}$

The reproduction number for drug-sensitivity is the largest Eigen value, that is

$\mathrm{R}_{\mathrm{os}}=\rho\left(\mathrm{F} \mathrm{V}^{-1}\right) \frac{\mathrm{v}(1-\rho \mathrm{S}) \beta \mathrm{S} \pi(\theta+\mu-\mu \sigma)}{\mu(\theta+\mu)(\mathrm{v}+\mu)(\mu+\mu \mathrm{T}+r 2)}+\frac{\rho \mathrm{S} \beta \mathrm{S} \pi(\theta+\mu-\mu \sigma)}{\mu(\theta+\mu)(\mathrm{v}+\mu)(\mu+\mu \mathrm{T}+r 2)}$

Local Stability of Disease Free Equilibrium Point with Drug-Sensitivity TB: Theorem 1: The disease free equilibrium point, $E_{o}$ is locally asymptotically stable if $\mathrm{R}_{\mathrm{os}}<1$ and unstable if $\mathrm{R}_{\mathrm{os}}>1$

Let

$\mathrm{F}_{1}=\sigma \pi-(\theta+\mu) M=0$

$\mathrm{F}_{2}=(1-\sigma) \pi+\theta M-(\beta S I S+\mu) S=0$

$\mathrm{F}_{3}=(1-\rho \mathrm{S}) \beta \mathrm{SISS}-(\mathrm{v}+\mu) E \mathrm{~S}=0$

$\mathrm{F}_{4}=\rho \mathrm{S} \beta \mathrm{SISS}+\mathrm{v} E \mathrm{~S}-(\mu+\mu \mathrm{T}+r 2) I \mathrm{~S}=0(46)$

$\mathrm{F}_{5}=r 2(1-\mathrm{r}) I S-\mu \mathrm{RS}=0$

Thus the Jacobean Matrix J for the system (43) to (47) is given by

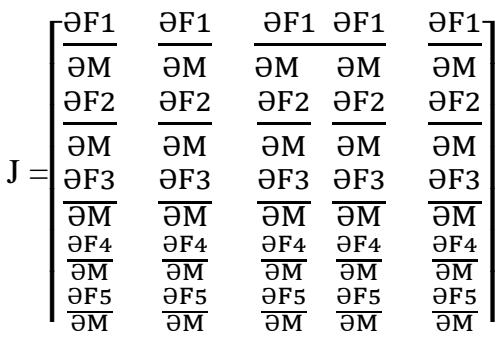

Substituting equation (43) to (47) into equation (48) and evaluating at the disease free equilibrium, we obtain

$$
\left.J\left(E_{0}\right)=\mid \begin{array}{cccccr}
-(\theta+\mu) & 0 & 0 & 0 & 0 \\
0 & -\mu & 0 & -\frac{\beta S \pi(\theta+\mu-\mu \sigma)}{\mu(\theta+\mu)} & 0 \\
0 & 0 & -(v+\mu) & \frac{(1-\rho S) \beta S \pi(\theta+\mu-\mu \sigma)}{\mu(\theta+\mu)} & 0 \\
& & & \frac{(1-\rho S) \beta S \pi(\theta+\mu-\mu \sigma)}{\mu(\theta+\mu)} & 0 \\
0 & 0 & \mathrm{v} & r 2(1-\mathrm{r}) & -\mu
\end{array}\right](49)
$$

Given

$\left|J\left(E_{0}\right)-\lambda I\right|=0$

Substituting equation (48) into equation (49), we obtain 


$$
\left[\begin{array}{cccccc}
-(\theta+\mu)-\lambda & 0 & 0 & 0 & 0 \\
0 & -\mu-\lambda & 0 & -\frac{\beta S \pi(\theta+\mu-\mu \sigma)}{\mu(\theta+\mu)} & 0 \\
0 & 0 & -(\mathrm{v}+\mu)-\lambda & \frac{(1-\rho \mathrm{S}) \beta \mathrm{S} \pi(\theta+\mu-\mu \sigma)}{\mu(\theta+\mu)} & 0 \\
0 & 0 & \mathrm{v} & \frac{(1-\rho \mathrm{S}) \beta \mathrm{S} \pi(\theta+\mu-\mu \sigma)}{\mu(\theta+\mu)}-\lambda & 0 \\
0 & 0 & 0 & r 2(1-\mathrm{r}) & -\mu-\lambda
\end{array}\right]=0(50)
$$

From equation (50), we observe that $\lambda_{1}=\mu_{1}, \lambda_{2}=\mu_{1}$ and $\lambda_{3}=-(\theta+\mu)$, thus equation (50) reduces to

$$
\left[\begin{array}{cc}
-(\mathrm{v}+\mu)-\lambda & \frac{(1-\rho S) \beta S \pi(\theta+\mu-\mu \sigma)}{\mu(\theta+\mu)} \\
\mathrm{v} & -(\mu+\mu \mathrm{T}+r 2)-\frac{\rho S \beta S \pi(\theta+\mu-\mu \sigma)}{\mu(\theta+\mu)}-\lambda
\end{array}\right]=0
$$

Now, equation (51) becomes

$\left[\begin{array}{cc}-d 1-\lambda & c 1 \\ v & -d 2-\lambda\end{array} \mid=0\right.$

Where

$d 1=v+\mu, d 2=(\mu+\mu \mathrm{T}+r 2)-\frac{\rho \mathrm{S} \beta \mathrm{S} \pi(\theta+\mu-\mu \sigma)}{\mu(\theta+\mu)}$ and $c 1=\frac{(1-\rho \mathrm{S}) \beta \mathrm{S} \pi(\theta+\mu-\mu \sigma)}{\mu(\theta+\mu)}$

From (52), we have $\mathrm{R}_{\mathrm{os}}$

$(-d 1-\lambda)(-d 2-\lambda)-v c 1=0$

$\lambda^{2}+d 1 \lambda+d 2 \lambda+d 1 d 2-v c 1=0$

$\lambda^{2}+(d 1+d 2)+d 1 d 2-v c 1=0$

$\rho 2 \lambda^{2}+\rho_{\mathrm{o}}=0$

Where: $\rho 2=1 ; \rho 1=d 1+d 2$ and $\rho_{\mathrm{o}}=d 1 d 2-\mathrm{v} c 1=(\mathrm{v}+\mu)(\mu+\mu \mathrm{T}+r 2) 1-\mathrm{R}_{\mathrm{os}}$

We apply Routh-Hurwitz criterion which states that all roots of the polynomials (54) have negative real part if and only if the coefficients $\rho_{\mathrm{I}}$, are positive and the determinant of the matrices $H_{\mathrm{I}}=\rho 1=d 1+d 2>0$ iff $d 1 d 2$ and

$\mathrm{H}_{2}=\left|\begin{array}{cc}\rho 1 & 0 \\ 1 & \rho_{\mathrm{o}}\end{array}\right|=\rho 1 \rho_{\mathrm{o}}=(d 1+d 2)(d 1 d 2-\mathrm{v} c 1)$

$=d 1^{2} d 2-d 1 v c 1+d 1 d 2^{2}-\mathrm{C} 2 v c 1=d 2^{2} d 2+d 1 d 2^{2}-v c 1(d 1+d 2)>0$ iff

$=d 1^{2} d 2+d 1 d 2^{2}>v c 1(d 1+d 2)$

Therefore, all the Eigen values of the polynomial (54) have negative real parts, implying that $\lambda_{2}<0$ and $\lambda_{5}<0$. Since all the value of $\lambda_{1}<0$, for $i=1,2,3,4,5$ when $\mathrm{R}_{\mathrm{os}}<$ 1 , we conclude that the disease-free equilibrium point is locally asymptotically stable.

Global Stability of Disease Free Equilibrium Point with Drug Sensitivity TB: The local dynamic of a general MSEIR model is determined by the reproduction number $R_{o s}$. If $R_{o s} \leq 1$, then each infected individuals in its entire period of infectiousness will produce less than one infected individuals on average. This means that the disease will be wiped out of the population. if $R_{o s}>1$, then each infected individuals in its entire infectious period having contact with susceptible individuals will produce more than one infected individuals implying that the disease persists in the population if $R_{o s}=1$ and this is defined as the disease threshold, then one individual infected one more individual. For $R_{o s} \leq 1$ the disease free equilibrium, is locally asymptotically stable while for $\mathrm{R}_{\text {os }}>1$ the disease free equilibrium becomes unstable. 
By using the theory of Lasalle-Lyapunov function V, we have will show the global asymptotic stability. The disease free equilibrium point is $\left(E_{S}, I_{S}\right)=(0,0)$.

Theorem 2: If $\mathrm{R}_{\mathrm{os}} \leq 1$, then the disease free equilibrium $\left(E_{S}, I_{S}\right)=(0,0)$ of the system is globally asymptotically stable on $\Omega$. We construct the following LasalleLyapunov function $\mathrm{V}\left(\mathrm{E}_{\mathrm{S}}, \mathrm{I}_{\mathrm{S}}\right)$ on the positively invariant compact set $\Omega$. Thus on $\Omega, \mathrm{V}\left(\mathrm{E}_{\mathrm{S}}, \mathrm{I}_{\mathrm{S}}\right)$ is continuous and non-negative. We define $\mathrm{V}\left(\mathrm{E}_{\mathrm{S}}, \mathrm{I}_{\mathrm{S}}\right)=$ $E_{S}+(v+\mu)$ Is. consider the system of ordinary differential equation given by:

$$
\begin{aligned}
& \frac{E \mathrm{~S}}{d t}=(1-\rho \mathrm{S}) \beta \mathrm{SISS}-(\mathrm{v}+\mu) E \mathrm{~S} \\
& \frac{I \mathrm{~S}}{d t} \rho \mathrm{S} \beta \mathrm{S} I \mathrm{~S} S+\mathrm{v} E \mathrm{~S}-(\mu+\mu \mathrm{T}+r 2) I \mathrm{~S}
\end{aligned}
$$

The above system can be written as

$$
\begin{aligned}
& \left(\begin{array}{c}
E S \\
I S
\end{array}\right)= \\
& {\left[\begin{array}{cc}
-(\mathrm{v}+\mu) & \frac{(1-\rho \mathrm{S}) \beta \mathrm{S} \pi(\theta+\mu-\mu \sigma)}{\mu(\theta+\mu)} \\
\mathrm{v} & \frac{\rho \mathrm{S} \beta \mathrm{S} \pi(\theta+\mu-\mu \sigma)}{\mu(\theta+\mu)}-(\mu+\mu \mathrm{T}+r 2)
\end{array}\right]\left(\begin{array}{c}
E S \\
I S
\end{array}\right)}
\end{aligned}
$$

Thus, equation (57) can be written as $I=A(I)$

$$
\begin{aligned}
& A=\left[\begin{array}{cc}
-(\mathrm{v}+\mu) & \frac{(1-\rho \mathrm{S}) \beta \mathrm{S} \pi(\theta+\mu-\mu \sigma)}{\mu(\theta+\mu)} \\
\mathrm{v} & \frac{\rho \mathrm{S} \beta \mathrm{S} \pi(\theta+\mu-\mu \sigma)}{\mu(\theta+\mu)}-(\mu+\mu \mathrm{T}+r 2)
\end{array}\right] \\
& \text { and } I=\left(\begin{array}{c}
E S \\
I S
\end{array}\right)
\end{aligned}
$$

If we define $\mathrm{V}^{\mathrm{T}}=(\mathrm{v}, \mathrm{v}+\mu)$, then the derivative along the trajectories is given by $\mathrm{V}=\mathrm{V}^{\mathrm{T}} A(I)$ as $\mathrm{V}^{\mathrm{T}} A(I)=(\mathrm{v}, \mathrm{v}+$

$$
\mu)\left[\begin{array}{cc}
-(\mathrm{v}+\mu) & \frac{(1-\rho \mathrm{S}) \beta \mathrm{S} \pi(\theta+\mu-\mu \sigma)}{\mu(\theta+\mu)} \\
\mathrm{v} & \frac{\rho \mathrm{S} \beta \mathrm{S} \pi(\theta+\mu-\mu \sigma)}{\mu(\theta+\mu)}-(\mu+\mu \mathrm{t}+r 2)
\end{array}\right]
$$

Simplifying equation (58), we have

$=(\mathbf{\square}(0 @(\mathrm{v}(1-\rho \mathrm{S}) \beta \mathrm{S} \pi(\theta+\mu-\mu \sigma)) /(\mu(\theta+$

$\mu))+(\mathrm{v}+\mu)(\rho \mathrm{S} \beta \mathrm{S} \pi(\theta+\mu-\mu \sigma)) /(\mu(\theta+$

$\mu))-(\mu+\mu \mathrm{T}+r 2)))$

$=(\mathbf{\square}(0 @(\mathrm{v}+\mu)(\mu+\mu \mathrm{T}+r 2)(\rho \mathrm{S} \beta \mathrm{S} \pi(\theta+\mu-$ $\mu \sigma)) /(\mu(\theta+\mu)(\mu+\mu \mathrm{T}+r 2))(\mathrm{v}(1-$ $\rho \mathrm{S}) \beta \mathrm{S} \pi(\theta+\mu-\mu \sigma)) /(\mu(\theta+\mu))))$ $=\left(\begin{array}{c}0 \\ (v+\mu)(\mu+\mu \mathrm{T}+r 2)(\operatorname{Ro}-1)\end{array}\right)$

Which is strictly decreasing when $\mathrm{R}_{\mathrm{os}}<1$. Thus, $V$ $\leq(v+\mu)(\mu+\mu \mathrm{T}+r 2)(\operatorname{RoS}-1)$. We define the set $E=\{(\mathrm{ES}, \mathrm{IS}) \in \Omega / V(\mathrm{ES}, \mathrm{IS})=0\}$. The largest invariant set is contained in the set $E$ for which $E S=0$ or IS $=0$. thus $V<0$ when $\mathrm{R}_{\mathrm{os}}>1$. If IS $=0$ or $\mathrm{R}_{\mathrm{os}}=1$,
$V=0$. Thus, by Lasalle's invariance principle the disease free equilibrium is globally asymptotically stable on $\Omega$.

Conclusion: This study presents a simple yet more realistic deterministic model for the transmission dynamics of tuberculosis. In contrast to many tuberculosis models in literature, we incorporated passive immunity, drug sensitive class to the first line of treatment for tuberculosis into the existing model by Waaler (1968). Analytical study was carried out using linearized stability and the results shows that the disease free equilibrium (DFE) points are locally asymptotically stable (LAS) whenever Ro < I and global asymptotically stable (GAS) whenever Ro $\leq 1$

\section{REFERENCES}

Abdul-halim, N. B. (2013). Tuberculosis Model: A Mathematical Analysis. Malaysia: University of Malaya, Kuala Lumpur.

Daniel, T. M. (2009). History of Tuberculosis. Respiratory Medicine, 100(6), 1862-1870.

Diekmann and JAP Heesterbeek, (2000). Mathematical Epidemiology of Infectious Diseases: Model Building, Analysis and Interpretation: $O$, Chichester: John Wiley pp. 303, ISBN 0-471-49241-8

Frauer, J., Denholm, J., McBryde, E. (2014). Construction of a Mathematical Model forTuberculosis Transmission in Highly Endemic RegionS of the Asia-Pacific. J. Theoretical Biol. 74-84.

Ibrahim, M. O, Ejieji, N. C; Egbetade, S. A. (2013). A mathematical Model for the Epidemiology of Tuberculosis with Estimate of the Basic Reproduction Number. Scholarly J. Mathematics and Computer Sci. 2(4), 40-45.

Semenza, J, Suk, J; Tsolova, S. (2010). Social Determinants of Infectious Diseases. A Public Hesith Priority Euro Surveill, 15(3), 2334-2347.

Semenza, J., Suk, J; Tsolova, S. (2010). Social Determinants of Infectious Diseases, A Public Health Priority E2.tro Surveill, 15, 1 12-127.

Trauer, J., Denholm, J., \& McBryde E.(2014).Construction of a Mathematical Model for Tuberculosis Transmission in Highly Endemic Regions of the Asia-Pacific. Journal of Theoretical Biologv, 74-84. 
Waaler,'H. (1968). The use of Mathematical Models in the Study of the Epidemiology of Tuberculosis. American J. Q/Public Health), 52(6), 1002-101 3.

WHO. (2014). Global Tuberculosis Report. Geneva: WHO Report.
WHO. (2015). Global Tuberculosis Report. Geneva: WHO Report.

WHO. (2016). Global tuberculosis report. Geneva

WHO. (2017). Global Tuberculosis Report. Geneva. 